\title{
O VAREJO NO BRASIL SOB A ÓTICA DO APOIO MULTICRITÉRIO À DECISÃO: RANKING, POSICIONAMENTO E RISCO DE SEUS SEGMNTOS
}

\author{
Frederico Silva Valentim Sallum \\ Origin Health Intelligence \\ Rua Irmã Gabriela, 51/319, São Paulo, SP \\ frederico.sallum@originhealth.com.br \\ Luiz Flavio Autran Monteiro Gomes \\ Ibmec RJ \\ Av. Presidente Wilson, 118/1110, Rio de Janeiro, RJ \\ luiz.gomes@ibmec.edu.br \\ Leonardo Silva Valentim Sallum \\ Centro Universitário IBMR \\ Av. das Américas, 2603, Rio de Janeiro, RJ \\ leonardosvsallum@gmail.com
}

\begin{abstract}
RESUMO
A Pesquisa Mensal do Comércio divulgada pelo IBGE tem por objetivo gerar indicadores para acompanhar o comportamento conjuntural do comércio varejista brasileiro e de seus principais segmentos. Nesta pesquisa, além de outros índices, é gerado o índice de comércio varejista ampliado por atividade que visa acompanhar a variação no volume de vendas dos principais segmentos econômicos em 12 unidades da Federação. O presente artigo busca analisar o índice do comércio varejista ampliado por atividade de agosto de 2017, que compara a variação do volume de vendas acumulada de janeiro até o mês do índice com igual período do ano anterior. Esta comparação permite gerar-se informações relevantes ao mercado através de seus resultados com a aplicação do método multicritério PROMÉTHÉE II. Assim, é possível associar-se aos segmentos econômicos estudados os seguintes elementos: um ranking dos segmentos, um cenário de posicionamento e um grau de risco comparativo dos mesmos. O método PROMÉTHÉE II identificou o segmento de Vestuário como o de melhor desempenho e o segmento de Papelaria com o de pior desempenho.
\end{abstract}

Palavra-chave: Varejo no Brasil; Apoio multicritério à decisão; Método PROMÉTHÉE II.

\begin{abstract}
The Monthly Commerce Survey disclosed by IBGE aims to produce indicators for following the conjunctural behavior of retailing and its main segments in Brazil. In this survey, besides other indexes, the retailing index thus generated is broadened by activity. This index follows the variation in the volume of sales of the main economic segments in 12 units of Brazil. The present article analyzes the retailing index broadened by activity as of August 2017. This last index compares the variation in sales volume accumulated from
\end{abstract}


January to August against the same period of the year before. This comparison allows generating relevant information for the market through applying the PROMÉTHÉE II method. It is therefore possible to associate to the economic segments the following elements: a ranking of the segments, a positioning scenario, and a degree of comparative risk. The PROMÉTHÉE II method identified the Clothing segment as that of worst performance and the Stationary segment as having the worst performance.

Keywords: Retailing in Brazil; Multicriteria decision aid; PROMÉTHÉE II method.

\section{Como Citar:}

SALLUM, F. S. V.; GOMES, L. F. A. M.; SALLUM, L. S. V. O varejo no Brasil sob a ótica do apoio multicritério à decisão: ranking, posicionamento e risco de seus segmentos. In: SIMPÓSIO DE PESQUISA OPERACIONAL E LOGÍSTICA DA MARINHA, 19., 2019, Rio de Janeiro, RJ. Anais [...]. Rio de Janeiro: Centro de Análises de Sistemas Navais, 2019.

\section{INTRODUÇÃO}

A Pesquisa Mensal do Comércio (PMC) é divulgada pelo IBGE (Instituto Brasileiro de Geografia e Estatística) com o objetivo de gerar indicadores para acompanhar o comportamento conjuntural do comércio varejista brasileiro e de seus principais segmentos (IBGE, 2017). Para isto, o IBGE busca investigar a receita bruta de revenda nas empresas formalmente constituídas, com 20 ou mais pessoas ocupadas, onde a atividade principal é o comércio varejista. Os indicadores gerados por esta pesquisa são de grande importância para mensurar o comportamento da atividade econômica. Além disso, estes indicadores oferecerem, mensalmente, um retrato do comportamento do comércio varejista brasileiro à população.

Acompanhar o comportamento do mercado varejista é de suma importância para gerar informações que apoiem a tomada de decisão de empresários, investidores e outros interessados nos segmentos do comércio. Sparemberger et al. (2017) citam que trabalhos onde se busca gerar informações sobre o setor varejista são de grande importância, dada a força que o comércio exerce na economia. Um dos índices gerados pela PMC é o índice de comércio varejista ampliado por atividade. Este índice é divulgado para alguns segmentos do varejo em 12 unidades da Federação, a fim de construir indicadores para duas variáveis: a receita nominal de vendas e o volume de vendas. São divulgados 4 tipos diferentes deste índice. Um deles é o índice acumulado do ano, que compara os índices acumulados nominais e de volume de receita bruta de revenda, de janeiro até o mês do índice com os de igual período do ano anterior (IBGE, 2017). Assim, o IBGE, através deste índice, apresenta mensalmente um retrato do desempenho de alguns segmentos econômicos, tornando possível aos interessados comparar o desempenho de diferentes segmentos no mercado varejista brasileiro.

Segundo Albuja et al. (2011) conhecer os segmentos mais atrativos para investir pode ser útil para todos os investidores. Estes autores também citam que o desempenho comparativo entre setores da economia ao longo do tempo e como empresas destacam-se em relação às outras em seu setor de atuação são questões estudadas como um dos temas centrais de pesquisas no campo de estudo de estratégia.

Assim sendo, este artigo objetiva construir um cenário que traduza a posição de cada segmento em relação aos demais no contexto estudado, estabelecer um ranking dos segmentos em relação ao seu desempenho nas 12 unidades da Federação estudadas e estabelecer um grau de risco comparativo para cada setor dentre os resultados de seus 
desempenhos. Para isto, será avaliado o desempenho de cada segmento econômico em relação aos demais através dos valores da variação do volume de vendas divulgado pela PMC de agosto de 2017 com uso do método PROMÉTHÉE II.

Em situações onde se deseja ordenar, selecionar, classificar ou descrever um conjunto de alternativas à luz de seus desempenhos em um conjunto de critérios, a aplicação de métodos multicritério de apoio à decisão vem sendo amplamente utilizada (VINCKE, 1989; ROY, 1991; SAATY, 1994; GOMES; GOMES, 2014). Nota-se neste artigo que os dados da PMC são divulgados em um contexto multicritério, pois são apresentados os valores da variação no volume de vendas dos segmentos econômicos nas unidades da Federação. Assim, cada segmento econômico é interpretado como sendo uma alternativa e as unidades da Federação como sendo os critérios. Portanto, o valor da variação no índice de cada segmento em cada unidade da Federação estudada representa o desempenho de cada alternativa em cada critério estudado.

O método PROMÉTHÉE II foi escolhido para desenvolver este estudo porque se baseia em uma comparação par a par entre o desempenho das alternativas critério por critério. Assim, este método é capaz de estabelecer uma relação de superação entre as alternativas mensurando em seu resultado com a devida sensibilidade a variação do desempenho destas entre os critérios.

$\mathrm{O}$ uso de um método multicritério torna-se útil quando se deseja estudar o comportamento de diferentes segmentos econômicos quando o desempenho de cada segmento pode variar dentre os estados brasileiros. Por exemplo, pode acontecer de algum segmento obter o melhor desempenho apenas em algum estado brasileiro, mas em outros não. Além disso, outros segmentos que possuem excelente desempenho na maioria dos estados podem apresentar baixo desempenho em outros. Sendo assim, nasce a necessidade de avaliar o desempenho dos segmentos econômicos através de uma metodologia que reflita em seus resultados com a devida sensibilidade a variação no desempenho de cada segmento estado por estado.

\section{REVISÃO BIBLIOGRÁFICA}

\subsection{MÉTODOS MULTICRITÉRIO DE APOIO À DECISÃO}

Os métodos multicritério de apoio à decisão buscam trazer auxílio a um processo decisório em que um conjunto de alternativas deve ser analisado simultaneamente por um conjunto de critérios que podem ser, por vezes, conflitantes (GOMES; ARAYA; CARIGNANO, 2004). A necessidade do ser humano de tomar decisões de forma organizada, com transparência, levando explicitamente em conta o risco e, frequentemente, na presença de um volume grande de informações, o leva à necessidade do uso de modelos de apoio à tomada de decisão (TZENG, HUANG, 2011).

Richardson (1999) salienta que o método é o conjunto das atividades sistemáticas e racionais que permitem alcançar o objetivo desejado. Ragsdale (2008) afirma que a construção de modelos é uma das formas mais eficientes para a escolha entre diversas alternativas através da análise e avaliação. Neste sentido, o método é o passo a passo a ser seguido para que cientistas possam obter auxílio em um processo decisório. Porém, vale a pena ressaltar que, por vezes, a ordem que diferentes partes do modelo são especificadas, a forma como os dados e preferências são coletados para aplicação de um método, dentre outras origens, podem causar dependência no resultado gerado pela aplicação de um método (HÄMÄLÄINEN; LAHTINEN, 2016). Por este motivo, deve-se conhecer as principais limitações de um método antes de usá-lo e buscar adaptação do contexto inserido no problema analisado ao passo a passo da metodologia a ser aplicada.

Desta forma, os métodos multicritério de apoio à decisão podem ser usados em 
situações de seleção, ordenação, classificação e descrição de um conjunto de alternativas com base em seus desempenhos em um conjunto de critérios. As duas grandes escolas que concentram estes métodos são: a Escola Francesa, também chamada Escola Europeia e a Escola Norte Americana de apoio multicritério à decisão (GOMES, 2007). Os métodos da Escola Francesa utilizam de uma comparação par a par entre alternativas a fim de estabelecer uma relação de superação entre elas. Os métodos da Escola Norte Americana buscam agregar todas as informações acerca do problema analisado por meio de uma grande síntese (RANGEL; GOMES, 2010).

Os principais métodos da Escola Francesa são os métodos ELECTRE (ROY; BOUYSSOU, 1993) e os métodos PROMÉTHÉE (BRANS; VINCKE; MARESCHAL, 1986). Como principais métodos da Escola Norte-Americana, pode-se citar o método AHP (Analytic Hierarchy Process) (SAATY, 1980) e a Teoria da Utilidade Multiatributo (Multiattribute Utility Theory) (KEENEY; RAIFFA, 1993). Também existem métodos multicritério designados híbridos: estes métodos não pertencem rigorosamente à Escola Francesa e nem à Escola Norte Americana, mas podem possuir elementos de ambas as escolas. Como exemplo de métodos híbridos, pode-se citar o método TOPSIS (Tecnique for order preference by similarity to Ideal Soluction) (HWANG; YOON, 1981), o método TODIM (Tomada de Decisão Interativa e Multicritério) (GOMES; LIMA, 1991) e o método MACBETH (Measuring Attractiveness by a Categorical Based Evaluation Technique) (BANA e COSTA; CORTE; VASNICK, 2012). Estes métodos podem possuir características de ambas as escolas em seu passo a passo.

Muitos estudiosos vêm aplicando estes métodos nos mais diferentes campos de estudo. No trabalho de Martins (2014) utilizou-se o método TOPSIS para ordenar um conjunto de 58 fundos de investimento multimercado e um conjunto de 27 fundos de investimento em ações analisando o desempenho de ambos os conjuntos em 19 critérios qualitativos e quantitativos. Com a aplicação do método TOPSIS foi possível estabelecer uma ordenação aos fundos multimercado e aos fundos de ações observando simultaneamente os desempenhos de cada fundo nos 19 critérios. A aplicação deste método facilitou a análise dos fundos para estabelecer uma ordenação. Pois, alguns fundos destacam-se apenas em alguns critérios e outros fundos nos demais critérios.

Pakizeh e Hosseini (2015) utilizaram o método PROMÉTHÉE II para analisar 4 projetos de venture capital baseado em seus desempenhos em 5 critérios. Estes autores ressaltam que o método PROMÉTHÉE II permitiu estabelecer uma avaliação detalhada ao avaliar os projetos em conjunto para uma posterior seleção, uma vez que este tipo de projeto possui alto risco de sucesso. Com a aplicação deste método cada critério de análise pôde ser modelado de acordo com as preferências dos envolvidos na seleção do projeto. Neste artigo, foi utilizado o método Entropy para gerar pesos aos critérios. Assim, segundo os autores, o método PROMÉTHÉE II tornou-se um sistema organizado para seleção de projetos de investimento.

Adali, Işik e Kundakci (2016) utilizaram o método TODIM para selecionar cursos eletivos para estudantes de graduação considerando, suas preferências e habilidades. Estes autores ressaltam em seu artigo a importância do adequado apoio à tomada de decisão sobre a escolha do curso, pois isto impacta diretamente na carreira profissional dos estudantes e em sua vida acadêmica. Estes autores, analisaram um conjunto de 6 cursos eletivos em um conjunto de 5 critérios. O método AHP foi utilizado para gerar peso a cada um dos critérios. Assim, foi possível ordenar as opções de cursos à luz de seus desempenhos no conjunto de critérios para posterior seleção de cada aluno.

No artigo de Yücel e Görener (2016) é relatado o caso em que uma companhia europeia de investimentos deseja adquirir uma empresa na Turquia. Esta companhia levantou um grupo de 4 empresas que considera comprar e, para tomar a decisão, 6 critérios precisam 
ser analisados. O método AHP foi utilizado para gerar pesos aos critérios. Em seguida, foi aplicado o método ELECTRE I. Este método possibilitou a análise do desempenho das 4 empresas em conjunto nos 6 critérios estabelecidos pela companhia europeia interessada em realizar a compra. Os autores deste artigo ressaltam que a aplicação do método ELECTRE I, além de permitir a análise simultânea das 4 empresas no conjunto de critérios, minimiza o risco de realizar um investimento errado. O método ELECTRE I selecionou 1 empresa para a companhia europeia realizar sua compra.

\subsection{O MÉTODO PROMÉTHÉE II}

Os métodos da família PROMÉTHÉE (Preference Ranking Organization Method for Enrichment Evaluations) pertencem à Escola Francesa de apoio multicritério à decisão. Existem 6 métodos pertencentes à família de métodos PROMÉTHÉE (I, II, III, IV, V e VI) onde cada um possui suas particularidades para a modelagem de acordo com as preferências do tomador de decisão. O método PROMÉTHÉE II, em particular, foi desenvolvido objetivando a ordenação de alternativas (BRANS; VINCKE; MARESCHAL, 1986).

Para isto, o método PROMÉTHÉE II busca estabelecer uma relação de superação entre alternativas. Então, faz-se em uma comparação entre pares de alternativas com base nas diferenças de desempenho critério por critério. Esta diferença é mensurada de forma recíproca entre os pares de alternativas, ou seja, a diferença no valor do desempenho que uma alternativa possui sobre a outra em um determinado critério é mensurado da mesma forma para ambas. Para a alternativa que possui melhor desempenho, mensura-se o valor desta diferença como parte do domínio que esta possui sobre as demais. Para a que possui pior desempenho, o valor desta diferença será mensurado como parte do quanto esta alternativa é dominada pelas demais.

É importante ressaltar que o método PROMÉTHÉE II possui limitações em sua aplicação. Estas limitações se resumem a reversões no ranking que podem ocorrer em determinados casos. Detalhes sobre estas limitações podem ser encontrados no artigo de Vetschera e Almeida (2012).

Os critérios estudados devem possuir o sentido de maximização ou minimização. Os critérios de maximização são os que quanto maior o desempenho da alternativa, melhor para obter a melhor posição na ordenação final. Para os critérios de minimização, acontece o contrário. Cada critério deve possuir seu peso de importância no processo decisório. A soma dos pesos de todos os critérios deve ser igual a 1 (Equação 1).

$\sum_{i=1}^{m} w_{j}=1$

Na Equação $1 w_{j}$ é o peso do critério $j$ em um conjunto de critérios $C$ que vai de 1 até $m$. Cada critério deve possuir sua função de preferência. A função de preferência é a forma de mensurar o valor da diferença do desempenho entre pares de alternativas. Os valores para mensurar estas diferenças variam de 0 até 1 . Assim, seja um conjunto de alternativas $A$ que vai de 1 até $n$, a função de preferência (valor da diferença de desempenho mensurado de 0 até 1) das alternativas $a_{1}$ e $a_{2}$ no critério $j$ é $P_{j}\left(a_{1}, a_{2}\right)$ e o valor da diferença do desempenho entre estas duas alternativas neste mesmo critério é dado por $d_{j}\left(a_{1}, a_{2}\right)$. O Quadro 1 apresenta as 6 funções de preferência dos métodos PROMÉTHÉE.

Quadro 1: Funções de Preferência dos Métodos PROMÉTHÉE

\begin{tabular}{|l|l|l|} 
Função de Preferência & Representação & Limiares \\
\hline
\end{tabular}




\begin{tabular}{|c|c|c|}
\hline Critério Usual & $P_{j}\left(a_{1}, a_{2}\right)=\left\{\begin{array}{l}0 \text { se } d_{j}\left(a_{1}, a_{2}\right) \leq 0 \\
1 \text { se } d_{j}\left(a_{1}, a_{2}\right)>0\end{array}\right.$ & Não Há \\
\hline Quase Critério & $P_{j}\left(a_{1}, a_{2}\right)=\left\{\begin{array}{l}0 \text { se } d_{j}\left(a_{1}, a_{2}\right) \leq q \\
1 \text { se } d_{j}\left(a_{1}, a_{2}\right)>q\end{array}\right.$ & q \\
\hline $\begin{array}{l}\text { Critério de } \\
\text { Preferência Linear }\end{array}$ & $P_{j}\left(a_{1}, a_{2}\right)=\left\{\begin{array}{c}0 \text { se } d_{j}\left(a_{1}, a_{2}\right) \leq 0 \\
\frac{d_{j}\left(a_{1}, a_{2}\right)}{p} \text { se } 0<d_{j}\left(a_{1}, a_{2}\right) \leq p \\
1 \text { se } d_{j}\left(a_{1}, a_{2}\right)>p\end{array}\right.$ & $\mathrm{p}$ \\
\hline Critério de Nível & $P_{j}\left(a_{1}, a_{2}\right)=\left\{\begin{array}{c}0 \text { se } d_{j}\left(a_{1}, a_{2}\right) \leq q \\
\frac{1}{2} \text { se } q<d_{j}\left(a_{1}, a_{2}\right) \leq p \\
1 \text { sed } d_{j}\left(a_{1}, a_{2}\right)>p\end{array}\right.$ & $\mathrm{q}, \mathrm{p}$ \\
\hline $\begin{array}{l}\text { Critério de Preferência Linear } \\
\text { com Zona de Indiferença }\end{array}$ & $P_{j}\left(a_{1}, a_{2}\right)\left\{\begin{array}{c}0 \text { se } d_{j}\left(a_{1}, a_{2}\right) \leq q \\
\frac{d_{j}\left(a_{1}, a_{2}\right)-q}{p-q} \text { se } q<d_{j}\left(a_{1}, a_{2}\right) \leq p \\
1 \text { se } d_{j}\left(a_{1}, a_{2}\right)>p\end{array}\right.$ & $\mathrm{q}, \mathrm{p}$ \\
\hline Critério Gaussiano & $P_{j}\left(a_{1}, a_{2}\right)\left\{\begin{array}{c}0 \text { se } d_{j}\left(a_{1}, a_{2}\right) \leq 0 \\
1-e^{\left(-\frac{d_{j}\left(a_{1}, a_{2}\right)^{2}}{2 \sigma^{2}}\right)} \text { se } d_{j}\left(a_{1}, a_{2}\right)>0\end{array}\right.$ & $\sigma$ \\
\hline
\end{tabular}

Fonte: Brans, Vincke e Mareschal (1986)

Observando o Quadro 1 pode-se notar as diferentes formas de mensurar a diferença de desempenho entre duas alternativas em um critério segundo cada função de preferência. $\mathrm{Na}$ função de preferência de critério usual, caso a diferença do desempenho entra as alternativas $a_{1}$ e $a_{2}$ seja positiva, o valor da função de preferência será de 1 para $a_{1}$ e 0 para $a_{2}$ no critério $j$. Caso seja menor ou igual a 0 , o valor da função de preferência de cada alternativa seria o contrário. Para as demais funções de preferência exige-se que o tomador de decisão escolha um limiar.

Na função de preferência Quase Critério, por exemplo, o tomador de decisão deve escolher um valor para o limiar $q$. Supondo que o valor de $q$ seja 3 em uma determinada situação, caso a diferença do desempenho entre $a_{1}$ e $a_{2}$ seja menor ou igual a 3 , o valor da função de preferência de $a_{1}$ sobre $a_{2}$ no critério $j$ será 0 . Caso o valor desta diferença fosse maior que 3 valor da função de preferência de $a_{1}$ sobre $a_{2}$ no critério $j$ seria 1 . Assim, é importante notar que os limiares de preferência são representados por $p$ e expressam o menor valor para $d_{j}\left(a_{1}, a_{2}\right)$, acima do qual existe uma preferência por $a_{1}$ em relação a $a_{2}$. Os limiares de indiferença são representados por $q$ e expressam o maior valor para $d_{j}\left(a_{1}, a_{2}\right)$, abaixo do qual existe uma indiferença na preferência por $a_{1}$ em relação a $a_{2}$. E assim conforme descrito no Quadro 1 para as demais funções de preferência.

No primeiro passo de uso do método PROMÉTHÉE II calcula-se o índice de preferência $\pi$ para todos os pares de alternativas (Equação 2), com base na função de preferência utilizada no critério e no peso do critério.

$$
\pi\left(a_{1}, a_{2}\right)=\sum_{j=1}^{k} w_{j} P_{j}\left(a_{1}, a_{2}\right)
$$

Na Equação 2, $\pi\left(a_{1}, a_{2}\right)$ é o índice de preferência entre as alternativas $a_{1}$ e $a_{2}$ no critério $j$ e $w_{j}$ é o peso do critério $j$. Este índice reflete a preferência de uma alternativa sobre todas as demais.

No segundo passo, calcula-se o fluxo de superação positivo $\phi^{+}$(Equação 3) e o 
fluxo de superação negativo $\phi^{-}$(Equação 4). O fluxo de superação positivo resulta dentro do intervalo $0 \leq \phi^{+} \leq 1$ expressando o quanto uma alternativa possui de domínio sobre as demais e o fluxo de superação negativo resulta dentro do intervalo $0 \leq \phi^{-} \leq 1$, expressando o quanto uma alternativa é dominada pelas outras.

$$
\phi^{+}\left(a_{1}\right)=\frac{1}{n-1} \sum_{a_{2}} \pi\left(a_{1}, a_{2}\right)
$$

Na Equação 3, $\phi^{+}\left(a_{1}\right)$ é o fluxo de superação positivo da alternativa a sobre a alternativa $a_{2}$ quando se utiliza o índice de preferência $\pi\left(a_{1}, a_{2}\right)$.

$$
\phi^{-}\left(a_{1}\right)=\frac{1}{n-1} \sum_{a_{2}} \pi\left(a_{2}, a_{1}\right)
$$

$\mathrm{Na}$ Equação 4, $\phi^{-}\left(a_{1}\right)$ é o fluxo de superação negativo da alternativa a sobre $a_{1}$ alternativa $a_{2}$ quando se utiliza o índice de preferência $\pi\left(a_{1}, a_{2}\right)$.

No terceiro passo, calcula-se o fluxo de superação líquido $\phi$ (Equação 5), diferença entre o fluxo de superação positivo e o fluxo de superação negativo. Com o resultado do fluxo de superação líquido faz-se a ordenação das alternativas.

$$
\phi\left(a_{1}\right)=\phi^{+}\left(a_{1}\right)-\phi^{-}\left(a_{1}\right)
$$

Na Equação 13, $\phi\left(a_{1}\right)$ é o fluxo de superação líquido da alternativa $a_{1}$.

\section{APLICAÇÃO DA METODOLOGIA}

\subsection{PESQUISA MENSAL DO COMERCIO DE AGOSTO DE 2017}

Este artigo busca comparar os segmentos econômicos divulgados na PMC de agosto de 2017 pelo IBGE através dos valores da variação do volume de vendas. Para isto, será utilizado o índice acumulado do ano, que compara a variação do volume de vendas acumulada de janeiro até o mês do índice com o de igual período do ano anterior a fim de conhecer o desempenho de cada segmento em relação aos demais. Desta forma, a pesquisa aqui constituída é descritiva e quantitativa, pois busca gerar informação adicional aos dados da PMC de agosto de 2017. De igual modo, descrever as características da PMC de forma mais clara a todos os interessados.

A variação do volume de vendas resulta da deflação dos valores nominais correntes por índices de preços específicos para cada grupo de atividade e para cada Unidade da Federação, construídos a partir dos relativos de preços do Índice Nacional de Preços ao Consumidor Amplo - IPCA (nova estrutura adotada a partir de janeiro de 2012) e do Sistema Nacional de Pesquisa de Custos e Índices da Construção Civil - SINAPI (IBGE, 2017). Na construção dos índices de preços das UFs não cobertas pelo IPCA, foram usados os relativos de preços da área geográfica mais apropriada (IBGE, 2017).

Para isto, será aplicado o método PROMÉTHÉE II, pois este método é capaz de realizar uma comparação entre pares de alternativas e estabelecer um ranking que reflita com a devida sensibilidade a variação do desempenho em cada segmento econômico dentre os estados da Federação estudados. Este estudo torna-se necessário porque o comportamento 
de cada segmento econômico não é o mesmo em todos os estados brasileiros. Assim, o uso de uma metodologia que gere informação com a devida sensibilidade a esta variação a partir dos dados divulgados pelo IBGE pode apoiar decisões importantes por todos que estão inseridos no comércio varejista brasileiro.

Desta forma, o objetivo desta pesquisa é conhecer qual segmento obteve a melhor posição em relação aos demais no mercado brasileiro e qual a colocação de cada um ao se estabelecer um ranking. Também se objetiva elaborar um cenário que traduza o comportamento dos segmentos econômicos de cada um em relação aos demais e associar um grau de risco a cada segmento econômico. $\mathrm{O}$ índice do comércio varejista ampliado por atividade (segmentos do varejo) divulga a variação do volume de vendas para 12 unidades da Federação: Rio Grande do Sul, Paraná, Santa Catarina, Rio de Janeiro, São Paulo, Minas Gerais, Espírito Santo, Bahia, Ceará, Pernambuco, Goiás e Distrito Federal. Os segmentos do varejo contidos nesta pesquisa são apresentados no Quadro 2 com uma abreviação para o nome de cada segmento a ser utilizada sempre que citados neste trabalho.

Quadro 2: Segmentos e sua Abreviação

\begin{tabular}{|c|c|}
\hline Segmento & Abreviação \\
\hline Combustíveis e lubrificantes & Combustíveis \\
\hline Supermercados, hipermercados, produtos alimentícios, bebidas e fumo & Supermercados \\
\hline Vestuário, calçados e tecidos & Vestuário \\
\hline Móveis e Eletrodomésticos & Móveis/Eletro \\
\hline Artigos farmacêuticos, médicos, ortopédicos e de perfumaria e \\
cosméticos & Farmácia \\
\hline Equipamentos e material para escritório, informática e comunicação & Escritório \\
\hline Livros, jornais, revistas e papelaria & Papelaria \\
\hline Outros artigos de uso pessoal e doméstico & Doméstico \\
\hline Veículos, motocicletas, partes e peças & Veículos \\
\hline Material de Construção & Construção \\
\hline
\end{tabular}

Fonte: IBGE

O Quadro 3 apresenta as classes do varejo contidas em cada segmento do

Quadro 2.

Quadro 3: Classes de cada Segmento

\begin{tabular}{|c|c|}
\hline Segmento & Classes \\
\hline Combustíveis & $\begin{array}{c}\text { Comércio varejista de combustíveis e veículos automotores; comércio } \\
\text { varejista de lubrificantes; }\end{array}$ \\
\hline $\begin{array}{c}\text { Supermercado } \\
\text { s }\end{array}$ & $\begin{array}{c}\text { Comércio varejista de mercadorias em geral, com predominância de } \\
\text { produtos alimentícios: hipermercados e supermercados, minimercados } \\
\text { mercearias e armazéns; comércio varejista de produtos alimentícios em geral } \\
\text { ou especializado em produtos alimentícios não especificados anteriormente: } \\
\text { produtos de fumo; comércio varejista de produtos de padaria, laticínio, doces, } \\
\text { balas e semelhantes; comércio varejista de carnes e pescados: açougues e } \\
\text { padarias; comércio varejista de bebidas; comércio varejista de } \\
\text { hortifrutigranjeiros; }\end{array}$ \\
\hline Vestuário & $\begin{array}{c}\text { Comércio varejista especializado em tecidos e artigos de cama, mesa e banho; } \\
\text { comércio varejista de artigos do vestuário e acessórios; comércio varejista de } \\
\text { calçados e artigos de viagem; }\end{array}$ \\
\hline Móveis/Eletro & $\begin{array}{c}\text { Comércio varejista especializado de eletrodomésticos e equipamentos de } \\
\text { áudio e vídeo; comércio varejista especializado de móveis, colchoaria e } \\
\text { artigos de iluminação; comércio varejista especializado de instrumentos } \\
\text { musicais e acessórios; comércio varejista de uso doméstico não especificado } \\
\text { anteriormente; comércio varejista de discos, CDS, DVDS e fitas; }\end{array}$ \\
\hline Farmácia & $\begin{array}{c}\text { Comércio varejista de produtos farmacêuticos para uso humano e veterinário; } \\
\text { comércio varejista de cosméticos, produtos de perfumaria e de higiene }\end{array}$ \\
\hline
\end{tabular}




\begin{tabular}{|c|c|}
\hline & pessoal; comércio varejista de artigos médicos e ortopédicos; \\
\hline Escritório & $\begin{array}{c}\text { Comércio varejista especializado de equipamentos e suprimentos de } \\
\text { informática; comércio varejista especializado de equipamentos de telefonia e } \\
\text { comunicação; }\end{array}$ \\
\hline Papelaria & Comércio varejista de livros, jornais, revistas e papelaria; \\
\hline Doméstico & $\begin{array}{l}\text { Comércio varejista de mercadorias em geral sem predominância de produtos } \\
\text { alimentícios; comércio varejista especializado de peças e acessórios para } \\
\text { aparelhos eletroeletrônicos para o uso doméstico, exceto informática e } \\
\text { comunicação; Comércio varejista de brinquedos e produtos recreativos; } \\
\text { comércio varejista de artigos de óptica; comércio varejista de artigos de } \\
\text { joalheria; comércio varejista de suvenires, bijuterias e artesanato; }\end{array}$ \\
\hline Veículos & $\begin{array}{l}\text { Comércio a varejo e por atacado de veículos automotores; representantes } \\
\text { comerciais e agentes do comércio de veículos automotores; comércio de peças } \\
\text { e acessórios para veículos automotores; comércio por atacado e a varejo de } \\
\text { motocicletas, peças e acessórios; representantes comerciais e agentes do } \\
\text { comércio de motocicletas, peças e acessórios; }\end{array}$ \\
\hline Construção & $\begin{array}{l}\text { Comércio atacadista de cimento; comércio atacadista de madeira e produtos } \\
\text { derivados; comércio atacadista de ferragens e ferramentas; comércio } \\
\text { atacadista de material elétrico; comércio atacadista de materiais de construção } \\
\text { não especificados anteriormente e de materiais de construção em geral; } \\
\text { comércio varejista de tintas e materiais para pintura; comércio varejista de } \\
\text { material elétrico; comércio varejista de vidros; comércio varejista de } \\
\text { ferragens, madeiras e materiais de construção; }\end{array}$ \\
\hline
\end{tabular}

Fonte: IBGE

O Quadro 4 apresenta a variação no volume de vendas dos segmentos econômicos em 12 estados da Federação divulgadas pela PMC de agosto de 2017.

Quadro 4: Variação do Volume de vendas no Comércio Varejista Ampliado por Atividade

\begin{tabular}{|c|c|c|c|c|c|c|c|c|c|c|c|c|}
\hline Segmento & CE & PE & BA & MG & ES & RJ & SP & PR & SC & RS & GO & DF \\
\hline Combustíveis & $-25,1$ & 4,2 & $-2,3$ & $-25,3$ & $-10,1$ & $-18,1$ & 12,9 & 17,3 & 3,0 & 11,1 & $-22,2$ & $-9,1$ \\
\hline Supermercados & $-1,5$ & $-1,4$ & $-13,0$ & 12,3 & $-13,3$ & $-2,8$ & $-2,3$ & 2,3 & 25,2 & 0,1 & $-11,5$ & $-13,9$ \\
\hline Vestuário & $-2,8$ & 17,0 & 6,3 & 31,8 & 12,4 & 5,1 & 9,3 & $-4,6$ & $-8,2$ & 24,6 & 0,2 & 0,1 \\
\hline Móveis/Eletro & $-14,5$ & 13,6 & 22,1 & 8,8 & 10,9 & 0,5 & 14,8 & $-3,4$ & 3,5 & 7,5 & 0,8 & 5,0 \\
\hline Farmácia & 10,9 & $-12,2$ & $-6,7$ & $-3,2$ & 7,8 & 2,1 & 3,3 & $-1,0$ & $-0,8$ & 0,6 & $-1,1$ & $-0,9$ \\
\hline Papelaria & $-16,8$ & $-22,4$ & 26,1 & 8,1 & $-12,6$ & $-5,2$ & $-0,6$ & $-14,6$ & 7,0 & $-20,5$ & $-13,4$ & $-4,9$ \\
\hline Escritório & 14,9 & 51,6 & 3,4 & $-27,8$ & 17,1 & $-18,2$ & $-9,3$ & 18,8 & 35,6 & 15,8 & $-28,1$ & 6,6 \\
\hline Doméstico & 5,1 & 5,7 & 4,7 & $-2,8$ & $-5,4$ & 2,0 & $-3,6$ & 1,0 & 4,8 & 5,2 & $-11,2$ & 1,1 \\
\hline Veículos & 3,3 & 5,2 & 0,9 & $-25,2$ & 25,2 & 2,9 & $-4,8$ & $-7,6$ & 11,3 & 18,2 & $-15,1$ & 19,9 \\
\hline Construção & 16,0 & $-13,6$ & 2,7 & $-1,0$ & $-26,0$ & 10,9 & 13,3 & 14,7 & 1,0 & 2,4 & $-6,4$ & 24,9 \\
\hline
\end{tabular}

Fonte: IBGE

\subsection{APLICAÇÃO DO MÉTODO PROMÉTHÉE II}

A aplicação de um método multicritério justifica-se neste trabalho pela forma como os dados da variação do volume de vendas são apresentados pela PMC, pois os dados são divulgados em um contexto multicritério. Neste contexto, as alternativas são os segmentos econômicos e os critérios são as 12 unidades da Federação. A variação do volume de vendas de cada segmento em cada uma destes 12 estados brasileiros é o desempenho das alternativas nos critérios (Quadro 4). E, assim, deseja-se saber qual alternativa apresenta o melhor desempenho frente às demais e qual a posição de cada uma em relação às outras. Desta forma, a os dados utilizados nesta pesquisa são os dados da PMC de agosto de 2017 (Quadro 4) divulgados pelo IBGE (2017).

Na aplicação do método PROMÉTHÉE II dá-se igual peso a todos os critérios, pois se entende que todos os estados possuem igual importância na variação do volume de vendas. Todos os critérios são de maximização, pois a melhor alternativa será a capaz de apresentar a maior variação no volume de vendas de todos os estados estudados 
simultaneamente quando comparadas com as demais alternativas. A função de preferência de todos os critérios será o critério de preferência linear. Para determinar o limiar de preferência a ser utilizado, deverá calcular-se a diferença entre a maior e menor variação do volume de vendas de cada critério (Equação 6). Após isto, o critério que apresentar maior diferença entre sua maior e menor alternativa será o critério escolhido para gerar o limiar $p$ de todos os critérios, ou seja, o valor da maior diferença será o limiar $p$ de todos os critérios.

$$
p=d_{j}^{+}=a_{j}^{+}-a_{j}^{-}
$$

Na Equação 6, $p$ é o limiar de preferência em todos os critérios, $d_{j}^{+}$é o valor da maior diferença entre a maior e menor variação do volume de vendas em um mesmo critério $(j), a_{j}^{+}$e $a_{j}$ são os valores do maior e menor desempenhos em um mesmo critério $(j)$. Como o valor da função de preferência varia entre 0 e 1 , quando forem calculadas todas as diferenças, o valor da maior diferença terá o maior valor possível da função de preferência e os valores inferiores de uma diferença qualquer entre o desempenho de 2 duas alternativas ocorrerá de forma proporcional.

Para o cálculo do método PROMÉTHÉE II será utilizado o software Visual PROMETHHE ${ }^{\circledR}$. Assim, os dados contidos no Quadro 4, matriz de decisão multicritério aqui estudada, devem ser inseridos no software. Como todos os critérios possuem igual peso, o peso de cada critério será 1/12. Após calcularem-se as diferenças entre os valores de desempenho máximo e mínimo em cada critério, seleciona-se o valor da maior diferença para ser o limiar de preferência de todos os critérios. Após isto, o valor do limiar de preferência de todos os critérios é 74,0.

\subsubsection{Análise de Dados}

\subsubsection{Ordenação e Cenário dos Segmentos Econômicos}

A Figura 1 apresenta o ranking obtido pelo método PROMÉTHÉE II para os segmentos econômicos estudados.

Figura 1: Ranking dos Segmentos Econômicos

\begin{tabular}{|c|l|r|r|r|r|}
\hline Rank & \multicolumn{2}{|c|}{ action } & Phi & Phi+ & Phi- \\
\hline $\mathbf{1}$ & Vestuário & $\square$ & 0,0933 & 0,1529 & 0,0596 \\
\hline $\mathbf{2}$ & Escritório & $\square$ & 0,0798 & 0,1813 & 0,1015 \\
\hline $\mathbf{3}$ & Móveis/Eletro & $\square$ & 0,0663 & 0,1261 & 0,0599 \\
\hline $\mathbf{4}$ & Construção & $\square$ & 0,0279 & 0,1220 & 0,0941 \\
\hline $\mathbf{5}$ & Veículos & $\square$ & 0,0220 & 0,1107 & 0,0888 \\
\hline $\mathbf{6}$ & Doméstico & $\square$ & $-0,0126$ & 0,0683 & 0,0809 \\
\hline $\mathbf{7}$ & Farmácia & $\square$ & $-0,0223$ & 0,0744 & 0,0967 \\
\hline $\mathbf{8}$ & Supermercados & $\square$ & $-0,0456$ & 0,0749 & 0,1205 \\
\hline $\mathbf{9}$ & Combustíveis & $\square$ & $-0,1005$ & 0,0569 & 0,1575 \\
\hline $\mathbf{1 0}$ & Papelaria & $\square$ & $-0,1082$ & 0,0618 & 0,1700 \\
\hline
\end{tabular}

Fonte: Software Visual PROMETHEE ${ }^{\circledR}$

Observando a Figura 1, phi é o fluxo de superação líquido, ph+ é o fluxo de superação positivo e ph- é o fluxo de superação negativo. Pode-se observar que o segmento de vestuário obteve o melhor desempenho dentre os outros segmentos, ou seja, possui a maior variação no volume de vendas no índice estudado de janeiro até agosto de 2017 
comparado com igual período do ano anterior quando comparado aos demais segmentos critério por critério. Esta ordenação reflete o desempenho de todos os segmentos quando comparados um com os outros em todos os critérios. A Figura 2 apresenta o cenário de posicionamento dos segmentos estudados pelo método PROMÉTHÉE II.

Figura 2: Cenário de Posicionamento dos Segmentos Econômicos

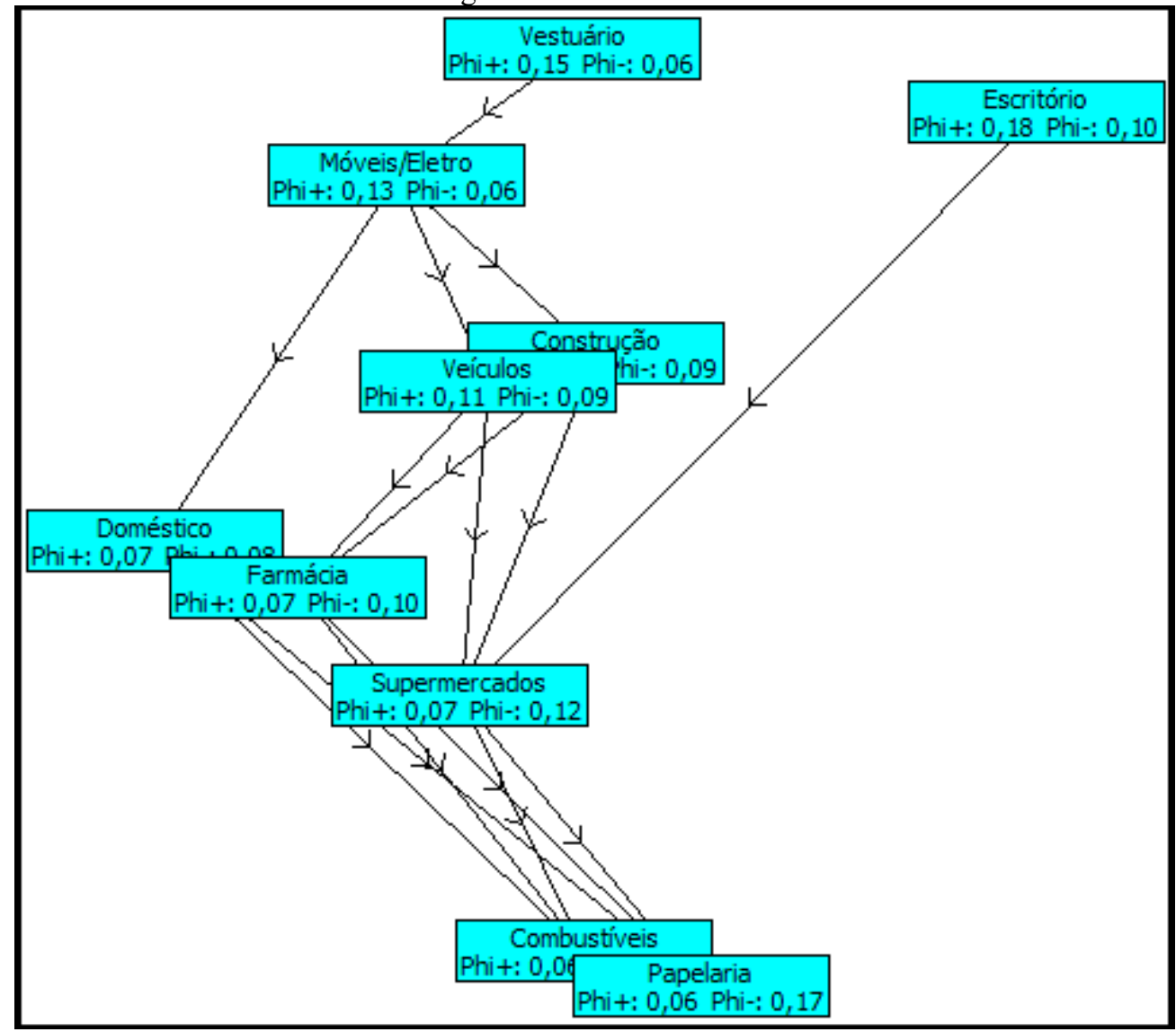

Fonte: Software Visual PROMETHEE ${ }^{\circledR}$

No cenário apresentado na Figura 2, pode-se observar a distância entre os segmentos segundo seus desempenhos. O cenário é a tradução de toda a matriz multicritério estudada (Quadro 4). Este cenário visa trazer clareza à compreensão do comportamento da variação do desempenho dos segmentos nas 12 unidades da Federação estudadas. A visualização do cenário complementa a ordenação, pois mostra em que distância se encontra uma posição da outra.

\subsubsection{Grau de Risco dos Segmentos Econômicos}

Com a aplicação do método PROMÉTHÉE II, também se busca atribuir a cada segmento econômico um grau de risco baseado na comparação de desempenho entre os segmentos. Este grau de risco varia de A até E. Estas classificações são atribuídas com base no valor do fluxo de superação líquido obtido pelo método PROMÉTHÉE II (Quadro 5).

Quadro 5: Classificações de Risco

\begin{tabular}{|c|c|}
\hline Fluxo de Superação Líquido ( $\phi)$ & Grau de Risco \\
\hline$[0,5<\phi \leq 1]$ & $\mathrm{A}$ \\
\hline$[0<\phi \leq 0,5]$ & $\mathrm{B}$ \\
\hline$[-0,5<\phi \leq 0]$ & $\mathrm{C}$ \\
\hline$[-1<\phi \leq-0,5]$ & $\mathrm{D}$ \\
\hline$[\phi=-1]$ & $\mathrm{E}$ \\
\hline
\end{tabular}


Fonte: Elaborado pelos Autores

Observando o Quadro 5, nota-se que o grau de risco E será atribuído somente no caso de algum segmento obtiver fluxo de superação líquido igual a -1, ou seja, para receber a pior classificação de risco é necessário possuir a pior variação no volume de vendas em todos os critérios. As demais classificações são atribuídas em intervalos de 0,5 entre os valores do maior e menor fluxo de superação líquido possíveis na aplicação do método PROMÉTHÉE II.

Vale a pena ressaltar que o grau de risco aqui atribuído é comparativo. Ou seja, para estabelecer a classificação de risco de um determinado segmento, esta classificação depende do desempenho de todos os segmentos em comparação ao desempenho do segmento em questão. A Figura 3 apresenta o grau de risco atribuído aos segmentos econômicos.

Figura 3: Grau de Risco Atribuído aos Segmentos Econômicos

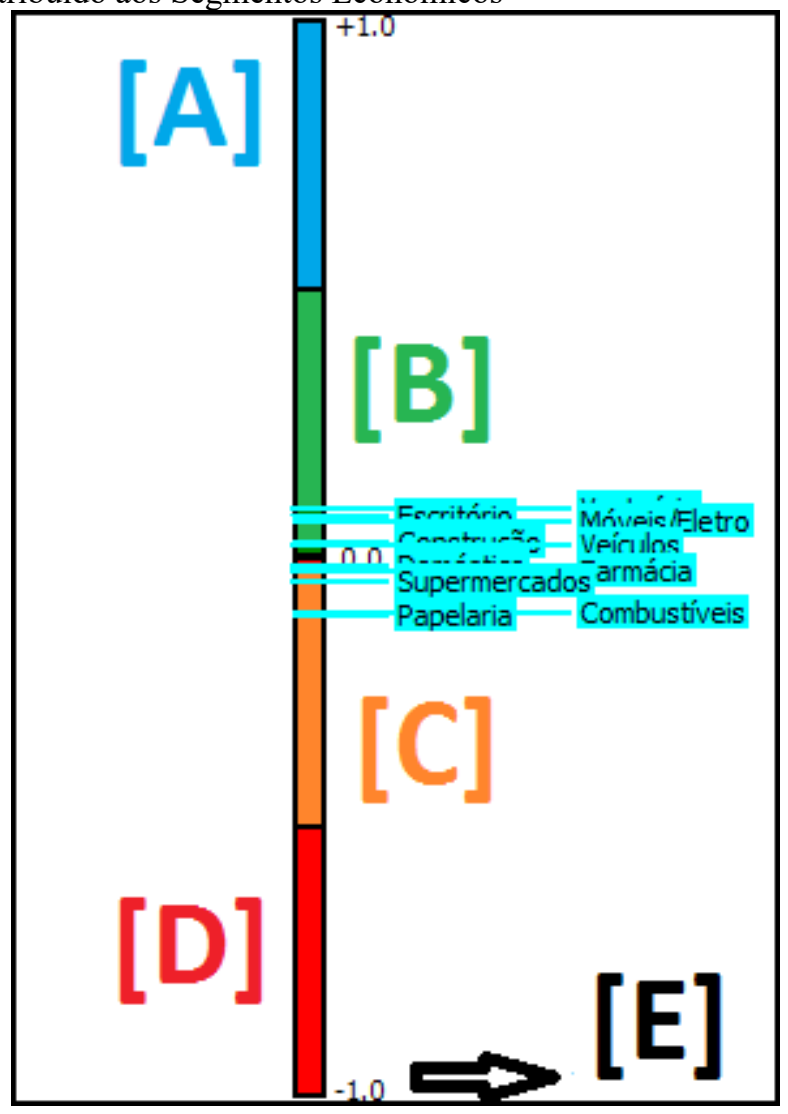

Fonte: Editado de Software Visual PROMETHEE ${ }^{\circledR}$

Como os segmentos encontram-se em com valores muito próximos nas classificações B e C, o Quadro 6 descreve o valor do fluxo de superação líquido, a ordenação e o grau de risco de cada segmento.

Quadro 6: Ordenação e Grau de Risco dos Setores Econômicos

\begin{tabular}{|c|c|c|c|}
\hline Ordem & Segmento Econômico & Fluxo de Superação Líquido & Grau de Risco \\
\hline $1^{\mathbf{o}}$ & Vestuário & 0,0933 & $\mathrm{~B}$ \\
\hline $2^{\mathbf{o}}$ & Escritório & 0,0798 & $\mathrm{~B}$ \\
\hline $3^{\mathbf{o}}$ & Móveis/Eletro & 0,0663 & $\mathrm{~B}$ \\
\hline $4^{\mathrm{o}}$ & Construção & 0,0279 & $\mathrm{~B}$ \\
\hline $5^{\mathbf{o}}$ & Veículos & 0,0220 & $\mathrm{~B}$ \\
\hline $6^{\mathbf{o}}$ & Doméstico & $-0,0126$ & $\mathrm{C}$ \\
\hline
\end{tabular}




\begin{tabular}{|c|c|c|c|}
\hline $7^{\circ}$ & Farmácia & $-0,0223$ & $\mathrm{C}$ \\
\hline $8^{\circ}$ & Supermercados & $-0,0456$ & $\mathrm{C}$ \\
\hline $9^{\circ}$ & Combustíveis & $-0,1005$ & $\mathrm{C}$ \\
\hline $10^{\circ}$ & Papelaria & $-0,1082$ & $\mathrm{C}$ \\
\hline
\end{tabular}

Fonte: Elaborado pelos Autores

Observando a Figura 3, é possível perceber que nenhum segmento obteve grau de risco $\mathrm{E}$ e todos possuem classificação de risco $\mathrm{B}$ ou $\mathrm{C}$. Ou seja, todos os segmentos apresentaram desempenho superior a pelo menos algum outro segmento em todos os critérios. Nenhum segmento apresentou o pior desempenho em todos os critérios. Nenhum segmento apresentou grau de risco A, ou seja, nenhum segmento se destaca de maneira forte o suficiente perante os demais. Este grau de risco também traz clareza ao entendimento sobre como se comportam os segmentos. Por exemplo, pode-se notar que o segmento Vestuário, apesar de apresentar o primeiro lugar na ordenação (Figura 1), não possui classificação de risco A (Quadro 6). Ou seja, trata-se de um primeiro lugar que não possui grande distância dos demais. Além disto, deve-se ressaltar que o segmento Vestuário possui a mesma classificação de risco de outros segmentos que se encontram em posições inferiores à sua (Quadro 6).

Também é possível notar que o setor Papelaria, apesar de obter o último lugar na ordenação, possui a mesma classificação de risco de outros setores que se encontram em posição superior à sua. Isto demonstra que, apesar deste setor obter o último lugar na ordenação, não se encontra tão distante dos que estão acima e nem é mais arriscado que estes. A classificação de risco aufere sensibilidade à ordenação apresentando até que ponto um setor é mais atrativo que outro.

\section{CONCLUSÃO}

O presente artigo analisou a variação do volume de vendas acumulado do índice do comércio varejista ampliado por atividade. Este índice corresponde ao período de janeiro a agosto de 2017 com o acumulado de igual período do ano anterior de 10 segmentos econômicos em 12 unidades da Federação divulgados na Pesquisa Mensal do Comércio. A principal motivação para tal análise é conhecer o segmento com melhor desempenho frente os demais e qual a posição de cada setor em comparação com os outros.

Para desenvolver esta análise foi utilizado o método multicritério PROMÉTHÉE II. Fez-se a escolha de um método multicritério para desenvolver esta análise porque os dados divulgados pelo IBGE encontram-se em um contexto multicritério. Neste contexto, os setores econômicos são o conjunto de alternativas que se deseja analisar, os critérios são as 12 unidades da Federação utilizadas nesta parte da PMC e o desempenho das alternativas nos critérios são os valores da variação do volume de vendas de cada setor em cada um dos 12 estados brasileiros estudados.

A aplicação de um método multicritério também se justifica nesta análise porque as variações nos volumes de vendas dos setores variam de estado para estado. Ou seja, o setor que obtém o melhor desempenho em um determinado estado pode não possuir este desempenho em outros estados. Desta forma, a aplicação do PROMÉTHÉE II baseada em uma comparação entre pares de alternativas critério por critério reflete esta questão com a devida sensibilidade em seu resultado.

A aplicação do método PROMÉTHÉE II gerou novas informações através dos dados divulgados pelo IBGE, como um ranking dos segmentos nos critérios estudados. Além disso, foi gerado um cenário de posicionamento para os segmentos em análise e um grau de risco comparativo para cada segmento. Foi possível notar durante a análise que o setor posicionado em primeiro lugar no ranking possui o mesmo grau de risco de outros 
segmentos que se encontram em posições inferiores a ele. Da mesma forma que o setor posicionado em último lugar no ranking estabelecido pelo método PROMÉTHÉE II possui a mesma classificação de risco de outros setores que se encontram em posição superior a ele.

Desta forma, a principal contribuição gerada por este artigo é apresentar um modelo capaz de expressar com maior clareza os dados do comportamento do comércio varejista brasileiro divulgados na Pesquisa Mensal do Comércio. Esta clareza reflete-se no cenário de posicionamento, no ranking e no grau de risco comparativo para os segmentos estudados descritos ao longo do artigo. Este modelo torna-se útil uma vez que gestores devem conhecer o comportamento do segmento de seu negócio em relação aos demais segmentos para formulação de estratégias e apoio à tomada de decisão. As limitações do modelo são referentes ao uso do método PROMÉTHÉE II com possíveis reversões no ranking geradas por este método. Todo gestor deve conhecer estas limitações para uma aplicação consciente do modelo.

Para pesquisas futuras, sugere-se a aplicação do método PROMÉTHÉE II aos outros índices divulgados na PMC assim como em outros tipos de índices. Também é interessante como contribuição a esta pesquisa a aplicação de outros métodos multicritério à PMC, com a devida justificativa do uso da metodologia desses outros métodos.

\section{REFERÊNCIAS BIBLIOGRÁFICAS}

[1] ADALI, E. A.; IŞIK, A. T.; KUNDAKCI, N. Todim method for the selection of the elective courses. European Scientific Journal, special edition, p. 314-324, 2016.

[2] ALBUJA, C. D.; GARCIA, F. G.; MOREIRAS, L. M. F.; TAMBOSI FILHO, E. Onde investir nos BRICS? Uma análise sob o prisma da organização industrial. Revista de Administração de Empresas, v. 51, n. 4, p. 349-369, 2011.

[3] BANA e COSTA, C. A.; CORTE, J. M.; VASNICK, J. C. MACBETH. International Journal of Information Technology \& Decision Making, v. 11, n. 2, p. 359-387, 2012.

[4] BRANS, J. P.; VINCKE, P. H.; MARESCHAL, B. How to select and how to rank projects: the PROMÉTHÉE methods. European Journal of Operational Research, v. 24, n. 2 , p. $228-238,1986$.

[5] GOMES, L. F. A. M. Teoria da decisão. São Paulo: Thompson, 2007.

[6] GOMES, L. F. A. M.; ARAYA, M. C. G.; CARIGNANO, C. Tomada de decisões em cenários complexos. São Paulo: Pioneira, 2004.

[7] GOMES, L. F. A. M.; GOMES, C. F. S. Tomada de decisão gerencial. 5. ed. São Paulo: Atlas, 2014.

[8] GOMES, L. F. A. M.; LIMA, M. M. P. P. TODIM: basics and application to multicriteria ranking of projects with environmental impacts. Foundations of Computing and Decision Sciences, v. 16, n. 3-4, p. 113-127, 1991.

[9] HÄMÄLÄINEN, R. P.; LAHTINEN, T. J. Path dependence in operational research how the modeling process can influence the results. Operations Research Perspectives, v. 3, p. 14-20, 2016.

[10] HWANG, C. L.; YOON, K. Multiple attribute decision making: methods and applications. Berlin: Springer-Verlag, 1981.

[11] IBGE. Instituto Brasileiro de Geografia e Estatística. Indicadores IBGE. Pesquisa Mensal de Comércio agosto de 2017. Disponível em: 
https://biblioteca.ibge.gov.br/visualizacao/periodicos/230/pmc_2017_ago.pdf. Acesso em: 30 out. 2017.

[12] KEENEY, R. L.; RAIFFA, H. Decisions with multiple objectives: preferences and value tradeoffs. Cambridge: Cambridge University Press, 1993.

[13] MARTINS, A. S. S. Seleção de fundos de investimento no Brasil: aplicação do método multicritério TOPSIS. 120 f. Dissertação (Mestrado Profissional em Administração) - Pós-Graduação e Pesquisa Ibmec, Faculdade de Economia e Finanças Ibmec, Rio de Janeiro, 2014.

[14] PAKIZEH, K.; HOSSEINI, M. Venture capital investment selection based on promethee. Applied Mathematics in Engineering, Management and Technology, v. 3, n. 1, p. 566-572, 2015.

[15] RAGSDALE, C. T. Spreadsheet modeling and decision analysis. Mason: Thomson, 2008.

[16] RANGEL, L. A. D.; GOMES, L. F. A. M. O apoio multicritério à decisão na avaliação de candidatos. Produção, v. 20, n. 1, p. 92-101, 2010.

[17] RICHARDSON, R. J. Pesquisa social: métodos e técnicas. São Paulo: Atlas, 1999.

[18] ROY, B. The outranking approach and the foundations of ELECTRE methods. Theory and Decisions, v. 31, p. 49-73, 1991.

[19] ROY, B.; BOUYSSOU, D. Aide multicritère à la décision: méthodes et cas. Paris: Econômica, 1993.

[20] SAATY, T. L. The Analytic Hierarchy Process. New York: Mc Graw Hill, 1980.

[21] SAATY, T. L. Fundamentals of decision making and priority theory: with the analytic hierarchy process. Pittsburgh: RWS Publications, 1994.

[22] SPAREMBERGER, A.; FROEMMING, L. M. S.; SIEDENBERG, D. R.; ZAMBERLAN, R.; CAPPELLARI, G. A confiança é capaz de propiciar estratégias competitivas para o setor varejista. Proyeciones UTN-BA, v. 15, p. 57-74, 2017.

[23] TZENG, G.; HUANG, J. Multiple attribute decision making: methods and applications. Boca Raton: CRC Press, 2011.

[24] VETSCHERA, R.; ALMEIDA, A. T. A PROMETHEE-based approach to portfolio selection problems. Computers \& Operations Research, v. 39, p. 1010-1020, 2012.

[25] VINCKE, P. L'aide multicritère à la dècision. Bruxelles: Éditions de l'Université de Bruxelles, 1989.

[26] YÜCEL, M. G.; GÖRENER, A. Decision making for company acquisition by ELECTRE method. International Journal of Suply Chain Management, v. 5, n. 1, p. 75-83, 2016. 